\title{
O.S.P.
}

L'orientation scolaire et professionnelle

$45 / 3 \mid 2016$

Varia

\section{Les tests BV9 et B53 peuvent-ils prédire la réussite scolaire?}

Can the B53 and BV9 tests predict the academic achievement?

\section{Alexandre Aubry et Béatrice Bourdin}

\section{(2) OpenEdition}

\section{Journals}

Édition électronique

URL : http://journals.openedition.org/osp/5070

DOI : 10.4000/osp.5070

ISSN : 2104-3795

Éditeur

Institut national d'étude du travail et d'orientation professionnelle (INETOP)

\section{Édition imprimée}

Date de publication : 15 septembre 2016

ISSN : 0249-6739

\section{Référence électronique}

Alexandre Aubry et Béatrice Bourdin, «Les tests BV9 et B53 peuvent-ils prédire la réussite scolaire ? », L'orientation scolaire et professionnelle [En ligne], 45/3 | 2016, mis en ligne le 01 septembre 2018,

consulté le 16 décembre 2020. URL : http://journals.openedition.org/osp/5070 ; DOI : https://doi.org/ $10.4000 /$ osp. 5070

Ce document a été généré automatiquement le 16 décembre 2020.

(C) Tous droits réservés 


\title{
Les tests BV9 et B53 peuvent-ils prédire la réussite scolaire?
}

\author{
Can the B53 and BV9 tests predict the academic achievement?
}

\author{
Alexandre Aubry et Béatrice Bourdin
}

\section{Introduction}

La relation entre l'intelligence et les performances scolaires est bien documentée depuis plusieurs décennies (Gagné \& St Père, 2002; McGrew \& Wendling, 2010; Walberg, 1984). L'évaluation de l'intelligence permet de prédire la réussite à l'école (Jensen, 1998 ; Kuncel, Hezlett, \& Ones, 2004). Pourtant, le lien entre l'intelligence et les notes scolaires n'est pas parfait. Dans la littérature, la force du lien varie entre modérée et forte, $\mathrm{r}$ 閎 $[.40 ; .70]$ (Mackintosh, 2004). Selon les études, environ $50 \%$ de la variance des performances scolaires serait expliquée par le facteur général de l'intelligence (Luo \& Petrill, 1999 ; Rohde \& Thompson, 2007). Cette relation est influencée par le contexte de l'environnement de l'apprentissage (Ceci, 1991), mais également par le type de mesure des performances scolaires utilisé dans les études. En général, elles sont évaluées avec des tests standardisés. La corrélation entre les capacités intellectuelles et les performances scolaires est habituellement plus élevée avec les tests standardisés qu'avec les notes scolaires (Mackintosh, 2004; Kaufman, Reynolds, Liu, Kaufman, \& McGrew, 2012). Peu de recherches examinent la liaison entre la notation scolaire et l'intelligence. Or, les notes scolaires paraissent cruciales dans l'orientation scolaire. Elles ont une influence sur la vie professionnelle future de l'élève (Roth, Bevier, Switzer, \& Schippmann, 1996). Les notes scolaires peuvent être considérées comme les performances effectives de l'élève (Rindermann \& Neubauer, 2004). Roth et ses collaborateurs (2015) ont réalisé une méta-analyse à partir de 162 études traitant la relation entre l'intelligence et les notes scolaires. Selon les auteurs, la mesure de l'intelligence est le meilleur prédicteur de la réussite scolaire. Les scores aux tests d'intelligence seraient modérément corrélés avec les notes scolaires, $\mathrm{r}=.54 ; 95 \% \mathrm{CI}$ [. $51 ; .67]^{1}$. D'après cette méta-analyse, les tests d'intelligence verbale, $r=.50 ; 95 \% \mathrm{CI}$ [. 
$48 ; .58]$, seraient plus liés aux performances scolaires que les tests non-verbaux, $\mathrm{r}=.44$; $95 \%$ CI $[.40 ; .47]$. De nombreuses recherches ont montré que certaines aptitudes cognitives peuvent expliquer, plus précisément, les performances scolaires au-delà de l'effet du facteur général de l'intelligence (Benson, 2008; Gustafsson \& Balke, 1993; Taub, Keith, Floyd, \& McGrew, 2008). Ces résultats montrent que l'intelligence n'est pas unidimensionnelle. Actuellement, la conception de l'intelligence est une représentation multifactorielle (Carroll, 1993), voire multidimensionnelle (Gardner, 1983) des aptitudes intellectuelles. Le modèle Cattell-Horn-Carroll $(\mathrm{CHC})$ est un des modèles issus de la conception multifactorielle de l'intelligence. Il est considéré comme un modèle psychométrique consensuel de la structure des aptitudes cognitives humaines (Schneider \& McGrew, 2012). Selon ce modèle, le potentiel intellectuel correspondrait à une organisation hiérarchique des aptitudes cognitives (voir Newton \& McGrew, 2010 pour une revue). Le modèle $\mathrm{CHC}$ s'appuie sur l'association de la théorie «Intelligence fluide - Intelligence cristallisée » de Cattell et Horn (Cattell, 1963 ; Horn \& Cattell, 1966) et du modèle « en trois strates » des capacités cognitives humaines de Carroll (1993). Le modèle $\mathrm{CHC}$ est organisé en trois strates hiérarchiquement ordonnées selon leur degré de généralité ou d'abstraction (Schneider, 2013a). La cime de toutes les aptitudes cognitives correspond au facteur général de l'intelligence (facteur $g$ ). Ce niveau élevé de généralité ou d'abstraction serait l'explication des corrélations positives entre les différentes épreuves dans une batterie d'évaluation des capacités cognitives (Carroll, 1991 ; Demetriou, Mouyi, \& Spanoudis, 2008 ; Jensen, 1998). Cependant, l'existence et la nature du facteur $g$ (Spearman, 1904) ne font pas actuellement consensus (Kvist \& Gustafsson, 2008 ; McGrew, 2009 ; Reynolds, Floyd, \& Niileksela, 2013 ; Van der Maas et al., 2006). D’un point de vue psychométrique, ce niveau cognitif élevé est souvent interprété comme une source latente de variance de plusieurs épreuves cognitives (Jensen, 1998; Reynolds et al., 2013; Schneider, 2013b). Le facteur $g$ est saturé de 16 aptitudes cognitives globales constituant la deuxième strate du modèle $\mathrm{CHC}$ (Schneider \& McGrew, 2012). Parmi ces facteurs de groupe, nous trouvons notamment les aptitudes de raisonnement fluide (Gf), de connaissance-compréhension (Gc), de mémoire à court terme (Gsm) et de vitesse de traitement (Gs). Cette taxonomie des aptitudes cognitives permet aux cliniciens de nuancer l'interprétation des scores à des batteries d'évaluation en matière de forces et de faiblesses (Schneider, 2013a; Warne, 2016). Chacune de ces aptitudes cognitives globales est composée d'environ 80 aptitudes spécifiques formant ainsi la troisième et dernière strate de ce modèle. Ces aptitudes cognitives spécifiques correspondraient à la variance spécifique des épreuves cognitives, alors que la variance partagée entre ces épreuves représenterait les aptitudes cognitives globales. Les aptitudes cognitives globales et spécifiques ne diffèrent pas fondamentalement. Ces deux types d'aptitudes cognitives se distinguent au niveau de la généralité des processus cognitifs impliqués. Par exemple, l'aptitude Gc recouvre notamment l'information verbale générale (Gc-K0), le développement langagier (Gc-LD) et la connaissance lexicale (Gc-VL) (Schneider \& McGrew, 2012). Le lien entre le facteur $g$ et les facteurs de groupe n'est pas homogène. Toutes les aptitudes cognitives globales n'ont pas la même saturation avec le facteur $g$ (Ackerman, Beier, \& Boyle, 2005). En effet, les aptitudes Gf et Gc sont plus saturées au facteur $g$ que les autres aptitudes cognitives (Bickley, Keith, \& Wolfle, 1995 ; Cornoldi, Orsini, Cianci, Giofrè, \& Pezzuti, 2013 ; Gignac, 2006 ; Golay, Reverte, Rossier, Favez, \& Lecerf, 2013 ; Keith, Fine, Taub, Reynolds, \& Kranzler, 2006 ; Parkin \& Beaujean, 2012). Actuellement, l'intelligence fluide fait référence à l'aptitude de raisonnement fluide (Gf). Elle est 
définie comme l'aptitude de raisonnement inductif et déductif nécessaire à la résolution de problèmes nouveaux. L'aptitude Gf est multidimensionnelle (Beauducel, Brocke, \& Liepmann, 2001). Le raisonnement inductif semble être au cœur de cette aptitude cognitive globale. Cette capacité cognitive générale permet l'acquisition de nouvelles compétences et de connaissances dans divers domaines. L'aptitude Gf et le facteur $g$ sont parfois confondus (Horn \& Blankson, 2005 ; Keith et al., 2006 ; Reynolds, Keith, Flanagan, \& Alfonso, 2013). Ce phénomène n'est pas consistant dans la littérature, du fait de l'hétérogénéité de l'échantillon utilisé dans les études (Kvist \& Gustafsson, 2008 ; Reynolds et al., 2013). Si l'échantillon est homogène, par exemple les participant.e.s ont tou.te.s le même niveau socio-économique, alors il y a une relation parfaite entre l'aptitude Gf et le facteur $g$. Classiquement, l'aptitude Gf est mesurée par des épreuves de raisonnement inductif où le.la participant.e. doit compléter une suite en sélectionnant un seul élément parmi plusieurs possibilités (Schneider, 2013a).

2 L'intelligence cristallisée a évolué vers le concept d'aptitude de connaissancecompréhension (Gc). Cette aptitude reflète les connaissances et les expériences acquises par l'individu, mais également l'application de ces connaissances (Newton \& McGrew, 2010). Elle est liée au développement des capacités langagières (Schipolowski, Wilhelm, \& Schroeders, 2014). L'aptitude Gc correspond à « une vaste étendue des connaissances et des compétences qui dépendent de la culture de l'individu " (Schneider \& McGrew, 2012, p. 122, notre traduction). L'aptitude Gc est fortement liée à la capacité de trouver une définition juste et adéquate d'un nouveau mot (Sternberg \& Powell, 1983). L'aptitude Gc serait un bon indicateur de la mobilisation de l'élève dans les apprentissages scolaires. Classiquement, l'aptitude Gc est mesurée par l'intermédiaire de tests d'intelligence évaluant les capacités de compréhension et de connaissances verbale, numérique ou figurale (Beauducel et al., 2001 ; Kan, Kievit, Dolan, \& Van der Maas, 2011). Habituellement, les aptitudes Gf et Gc sont modérément corrélées, $\mathrm{r}$ 撕 $[.48 ; .62]$ (Dang, Braeken, Ferrer, \& Liu, 2012; Friedman et al., 2006; Rindermann, Flores-Mendoza, \& Mansur-Alves, 2010; Colom \& Flores-Mendoza, 2007). L'aptitude Gf influence le déploiement de l'aptitude Gc grâce à l'investissement du raisonnement inductif dans les diverses situations d'apprentissage (Kvist \& Gustafsson, 2008). Ces deux aptitudes sont également fortement corrélées avec les performances scolaires. Pourtant, selon la récente métaanalyse de Postlethwaite (2011), l'aptitude Gf serait modérément corrélée aux performances scolaires, $\mathrm{r}=.40 ; 95 \% \mathrm{CI}[.35 ; .45]$. L'aptitude Gc serait plus fortement corrélée à la réussite scolaire, $\mathrm{r}=.65 ; 95 \% \mathrm{CI}[.63 ; .68]$. Elle serait un plus fort prédicteur des performances scolaires que l'aptitude Gf (Benson, 2008 ; Colom \& FloresMendoza, 2007; Flores-Mendoza et al., 2015). Cela signifierait que l'accumulation de connaissances apparaîtrait plus importante dans l'explication des performances scolaires. L'aptitude Gc médiatiserait les effets de l'aptitude Gf sur les performances (Schneider, 2013a). Un haut niveau de raisonnement permettrait d'acquérir plus de connaissances, permettant d'avoir un meilleur rendement scolaire. Ainsi, l'aptitude Gc faciliterait les apprentissages de connaissances nouvelles (Emmanuelli \& WeismannArcache, 2006).

3 Les psychologues exerçant dans les établissements du secondaire, c'est-à-dire les conseiller.ère.s d'orientation-psychologue (COP), sont parfois amené.e.s à examiner les freins de la réussite scolaire de certains élèves. Le but est d'apprécier le 
fonctionnement cognitif de l'élève par rapport à ses performances scolaires. Dans l'Académie d'Amiens, les COP réalisent systématiquement des évaluations psychométriques collectives dans les classes de troisième d'enseignement général. L'ambition de ce type d'évaluation est de mieux comprendre le fonctionnement intellectuel global de l'élève afin de l'accompagner dans son choix d'orientation scolaire. Il existe peu de mesures collectives de "l'intelligence générale » en modalité verbale. Deux outils sont souvent utilisés en psychologie de l'orientation (Bernaud, 2014) : le test B53 (Bonnardel, 1967) et le test BV9 (Bonnardel, 1966). Ils permettent d'obtenir une mesure de "l'intelligence générale " dans les modalités non verbales, pour le test B53, et verbale, pour le test BV9. Dans le cadre de l'orientation scolaire, l'intérêt de ces deux outils est la possibilité de réaliser les mesures en passation collective dans un délai d'une heure. Cependant, peu de données empiriques permettent d'établir un lien entre les évaluations avec les tests B53 et BV9 et les performances scolaires.

4 L'objectif de notre étude est donc d'analyser la relation prédictive des scores au B53 et au BV9 sur les résultats scolaires. Cet objectif nous permettra, ainsi, de discuter du lien entre les aptitudes Gf / Gc et la réussite à l'école.

\section{Méthode}

\section{Participant.e.s}

5 Quatre-vingt-un élèves âgés en moyenne de 14 ans et 6 mois ( \pm 6 mois) ont été évalués. Les participant.e.s, 39 garçons et 42 filles, sont tous des élèves de troisième d'enseignement général issus d'un collège public choisi aléatoirement. Il est constitué d'une proportion mixte au niveau des statuts socio-économiques. Les élèves sont regroupés en quatre classes. Les classes $\mathrm{C} 1$ et $\mathrm{C} 2$ sont principalement composées d'élèves appartenant à la section européenne. Ces élèves suivent des enseignements spécifiques d'approfondissement de la langue et de la culture anglaises. Ils ont deux heures de cours d'anglais supplémentaires par rapport aux deux autres classes. La classe $\mathrm{C} 3$ intègre un grand nombre d'élèves ayant pris l'option de découverte professionnelle de trois heures (DP3). Ce type d'option permet à l'élève de réaliser son projet professionnel en ayant des connaissances du monde professionnel. Dans cette option, l'élève découvre les métiers, le fonctionnement de l'entreprise et de son environnement socioéconomique. La classe $\mathrm{C} 4$ regroupe des élèves n'ayant aucune option spécifique en plus de leur emploi du temps classique.

\section{Matériel}

6 Test B53. Le test B53, élaboré par Bonnardel (1967), est utilisé pour évaluer le raisonnement inductif dans la modalité non verbale. Il permet d'apprécier la mise en œuvre des capacités intellectuelles dans la résolution de problèmes logiques (Nicollet, Guillen, Jouhar, \& Rossier, 2009). Ce test évaluerait l'aptitude de raisonnement fluide (Gf) dans la modalité non verbale. L'élève doit découvrir la règle générale de progression à partir d'une collection d'images se succédant. Il doit choisir l'élément qui complétera le mieux cette suite logique parmi un choix multiple. Le résultat de chaque participant.e correspond au nombre de réponses correctes dans une durée limitée à 
15 minutes. Le score peut aller de 0 à 60 . Cette épreuve comporte 65 items, dont 5 exemples permettant ainsi de s'assurer que l'élève a bien compris la tâche demandée. La difficulté de l'épreuve est croissante au fur et à mesure des items, cela afin que l'épreuve ait un fort pouvoir de discrimination des capacités de raisonnement des élèves. Selon Thiébaut (2000), la consistance interne est satisfaisante $(\alpha=.93)$.

7 Test BV9. Le test BV9, créé par Bonnardel (1966), est utilisé pour l'évaluation des capacités intellectuelles dans la modalité verbale. Il sature modérément le facteur général de l'intelligence (Juhel, 1993). Ce test est basé sur la capacité à résoudre des problèmes énoncés. Il fait intervenir les capacités de raisonnement impliquant les connaissances stockées en mémoire à long terme. Le vocabulaire utilisé est parfois désuet. Cela contraint l'intervention d'une bonne connaissance générale et demande de comprendre les items par le contexte. Par définition, ce test estimerait l'aptitude de compréhension-connaissance (Gc). L'aptitude Gf serait également impliquée, dans une moindre mesure, à cause du raisonnement logique requis dans la résolution de problème. Le BV9 se compose de 120 items se répartissant en différentes catégories et d'un exemple afin de s'assurer de la compréhension de l'élève face à la tâche demandée. Les diverses catégories sont présentes aléatoirement dans le test. Dans chaque catégorie, les items sont présentés selon un ordre de difficulté croissante. La durée est limitée à 45 minutes. La notation correspond à la somme des réponses réussies, avec un score maximal de 120. Dans la littérature, les performances scolaires sont estimées par une évaluation standardisée. En comparaison des tests standardisés des performances scolaires, les notes scolaires ont l'avantage de représenter des performances observées pertinentes. Par conséquent, nous avons pris les notes scolaires du premier trimestre de nos participant.e.s dans 8 disciplines différentes. Nous avons réalisé des regroupements afin d'améliorer la dispersion des performances scolaires entre les participant.e.s. Nous nous sommes basés sur la catégorisation des notes scolaires de Rindermann et Neubauer (2004) pour réaliser nos regroupements. Nous avons ainsi regroupé les 8 disciplines en 4 groupes : Arts (Éducation musicale et Arts plastiques), Langues (Français et Anglais), Maths-Sciences (Mathématiques, Sciences physiques et chimiques et Science de la vie et de la terre) et Sciences sociales (Histoire et Géographie). Nous avons également retenu la moyenne générale du premier trimestre que nous avons appelée moyenne scolaire générale (MSG). Les notes scolaires correspondent à la moyenne des différentes évaluations dans chaque discipline. La notation se réalise sur une échelle de 0 à 20 points (voir tableau 1).

TABLEAU 1. Moyennes et écarts-types de l'âge, des scores aux BV9 et B53 et des moyennes des notes scolaires par classe

\begin{tabular}{|l|l|l|l|l|}
\hline & Classe 1 (C1) & Classe 2 (C2) & Classe 3 (C3) & Classe 4 (C4) \\
\hline Âge & $14.29(.24)$ & $14.41(.38)$ & $14.67(.67)$ & $14.59(.66)$ \\
\hline B53 & $33.67(8.10)$ & $33.64(8.41)$ & $26.10(7.11)$ & $29.27(4.88)$ \\
\hline BV9 & $52.29(11.02)$ & $49.88(14.84)$ & $39.85(12.13)$ & $43.87(11.96)$ \\
\hline Sciences & $13.19(3.21)$ & $13.04(3.45)$ & $10.63(2.97)$ & $11.83(1.98)$ \\
\hline Langage & $13.76(2.51)$ & $12.41(3.33)$ & $10.92(2.69)$ & $12.09(2.12)$ \\
\hline
\end{tabular}




\begin{tabular}{|l|l|l|l|l|}
\hline Sciences sociales & $14.43(2.92)$ & $12.21(3.01)$ & $10.43(2.77)$ & $10.07(2.87)$ \\
\hline Arts & $13.90(2.14)$ & $13.87(1.91)$ & $12.43(1.75)$ & $13.66(2.65)$ \\
\hline Général & $13.76(2.40)$ & $13.22(2.42)$ & $11.35(2.34)$ & $12.32(1.74)$ \\
\hline
\end{tabular}

Note. Les écarts-types sont entre parenthèses. Sciences: Mathématiques, Sciences physiques et chimiques et Science de la vie et de la terre ; Langage : Français et Anglais ; Sciences sociales : Histoire-Géographie; Arts : Arts plastiques et Éducation musicale ; MSG : Moyenne scolaire générale au $1^{\mathrm{er}}$ trimestre.

TABLE 1. Means and standard deviations of age, BV9 and B53 scores and the average of the school grades per class

\section{Procédure}

Les évaluations psychométriques ont été réalisées collectivement, en un seul jour, par quatre COP. Une séance d'une heure et demie a été planifiée dans l'emploi du temps des élèves de troisième d'un même établissement choisi aléatoirement. Toutes les données sont normalement distribuées (voir tableau 1).

10 Les analyses statistiques ont été réalisées à l'aide du logiciel R 3.2.2 (R Core Team, 2015). Nous avons utilisé la librairie psych (Revelle, 2015) pour les analyses corrélationnelles et la librairie boot (Canty \& Ripley, 2015) pour la réalisation des ré-échantillonnages afin de déterminer les intervalles de confiance des différents paramètres statistiques. Nous avons également utilisé la librairie yhat (Nimon, Oswald, \& Roberts, 2013) pour les régressions multiples. Cette dernière librairie nous a permis d'analyser la part spécifique et commune de la variance des performances scolaires expliquée par nos deux prédicteurs, BV9 et B53 (Nimon, Lewis, Kane, \& Haynes, 2008).

\section{Résultats}

11 Nous observons un effet de la classe au niveau de la MSG, F( $3 ; 77)=4.391 ; \mathrm{MSE}=5.231$; $p<.01$. Nous trouvons le même effet au niveau des tests $\mathrm{B} 53, \mathrm{~F}(3 ; 77)=5.065$; $\mathrm{MSE}=55.86 ; p<.01$, et $\mathrm{BV} 9, \mathrm{~F}(3 ; 77)=4.027 ; \mathrm{MSE}=162.5 ; p<.05$. Les comparaisons posthoc utilisant les tests HSD Tukey indiquent que la MSG de la classe C3 $(M=11.35 ; E T=2.34)$ diffère significativement de celle des classes $C 1 \quad(M=13.76$; $E T=2.40)$ et $\mathrm{C} 2(M=13.22 ; E T=2.42)$. De même, le score moyen au BV9 de la classe C3 $(M=39.85 ; E T=12.13)$ est significativement plus faible que celui de la classe $C 1$ $(M=52.29 ; E T=11.02)$. Nous retrouvons également une différence significative entre le score moyen au B53 de la classe $C 3(M=26.10 ; E T=7.11)$ et celui des classes $\mathrm{C} 1$ $(M=33.67 ; E T=8.10)$ et $C 2(M=33.64 ; E T=8.41)$.

12 Les items des tests BV9 et B53 sont dichotomiques (réussite ou non-réussite) et de difficultés variées. Par conséquent, nous avons préféré une évaluation de la consistance interne avec la formule 20 de Kuder-Richardson (KR20). Cette méthode prend mieux en compte les difficultés des items ainsi que leurs variances. Cela nous permet de mettre en évidence la cohérence entre les items de chaque test. Nous estimons une consistance 
interne de .93 (KR20; $n=41$ participant.e.s) pour le test BV9 et de .87 (KR20; $n=41$ participant.e.s) pour le test B53.

Le tableau 2 représente l'ensemble des corrélations entre les performances scolaires et les scores aux BV9 et B53. Pour cette étude corrélationnelle, nous avons utilisé la méthode de Pearson, sachant que nous avons un échantillon de 81 élèves et que toutes nos données sont normalement distribuées. Nous observons très peu de différence au niveau des performances scolaires entre les différentes disciplines. Toutes les disciplines sont fortement corrélées à la MSG, $r_{(81)}=[.75 ; .97]$. Nous observons également une corrélation modérée à forte entre les scores aux tests BV9 et $\mathrm{B} 53, \mathrm{r}_{(81)}=$. $56 ; p<.01 ; 95 \% \mathrm{CI}[.38 ; .72]$. Environ $31,54 \%$ de la variance est commune aux deux tests. Les scores au BV9 sont plus fortement corrélés avec la MSG, $\mathrm{r}_{(81)}=.70 ; p<.01$; $95 \% \mathrm{CI}[.48 ; .80]$, que celles au B53, $\mathrm{r}_{(81)}=.50 ; p<.01 ; 95 \% \mathrm{CI}[.31 ; .65]$.

Les scores au B53 ont une variance commune comprise entre $12 \%$ à $23 \%$ avec les performances scolaires dans les différentes disciplines, $r_{(81)}=[.34 ; .48]$. Les performances au B53 semblent plus liées aux notes scolaires en sciences que celles des autres disciplines, $\mathrm{r}_{(81)}=.48 ; p<.01 ; 95 \%$ CI $[.26 ; .63]$. À l'inverse, les moyennes scolaires en arts sont plus faiblement corrélées avec les scores au B53 que celles des autres disciplines, $\mathrm{r}_{(81)}=.34 ; p<.05 ; 95 \% \mathrm{CI}[.13 ; .52]$. Globalement, les corrélations entre les performances scolaires et les scores au B53 sont toutes inférieures à celles avec les scores au BV9 (voir tableau 2).

Les scores au BV9 déterminent entre $14 \%$ et $52 \%$ de la variation des performances scolaires dans les disciplines, $\mathrm{r}_{(81)}=[.38 ; .72]$. Les moyennes scolaires en arts sont plus faiblement corrélées avec les scores au BV9 que celles des autres disciplines, $\mathrm{r}_{(81)}=.38$; $p<.05 ; 95 \%$ CI $[.14 ; .54]$.

16 Le lien entre les performances scolaires et les scores aux BV9 et B53 ne sont pas identiques selon le type de discipline (voir tableau 2). D'après l'ensemble de ces résultats, les performances scolaires semblent être plus liées à l'aptitude Gc qu'à l'aptitude Gf.

17 Nous avons ensuite réalisé des régressions multiples afin de déterminer la prédiction des résultats scolaires par les scores obtenus aux tests BV9 et B53. Les scores aux BV9 et B53 sont des variables non orthogonales, car ils sont corrélés entre eux, $\mathrm{r}_{(81)}=.56$; $p<.01$. Cela signifie que leur interaction aura indubitablement un effet sur l'analyse de régression. Nous avons alors calculé la variance unique et partagée des deux prédicteurs dans notre analyse de régression multiple. Le tableau 3 récapitule les résultats de ces différentes régressions multiples pour les différentes catégories de notes scolaires (maths-sciences, langues, science sociale et arts).

Les performances BV9 et B53 expliquent significativement la variance de la MSG, F(2 ; 78) $=4.12 ; p<.01 ; \mathrm{R}^{2}=.51 ; \mathrm{R}_{\text {ajusté }}^{2}=.49$. Comme pour l'analyse corrélationnelle, nous observons une différence de la part de variances des moyennes scolaires expliquées par les scores aux BV9 et B53 selon les disciplines. Les résultats au BV9 prédiraient plus les variations de moyennes dans la majorité des disciplines que les résultats au B53, notamment en langues (voir tableau 3). La variance partagée entre le BV9 et le B53 permet d'expliquer entre 9,2 \% et 21,7\% des performances scolaires dans les 4 types de disciplines scolaires. La variance unique du BV9 permet d'ajouter entre $5 \%$ et 37,1\% d'explication supplémentaire dans la variance des performances scolaires. À l'inverse, la variance unique du B53 n'ajoute peu ou aucune explication supplémentaire. 
TABLEAU 2. Intercorrélation entre les moyennes des notes scolaires des différentes matières et les scores aux BV9 et B53

\begin{tabular}{|c|c|c|c|c|c|c|c|}
\hline Variables & 1 & 2 & 3 & 4 & 5 & 6 & 7 \\
\hline 1. B53 & - & & & & & & \\
\hline 2. BV9 & $\begin{array}{l}.56^{* *}[.38, . \\
72]\end{array}$ & - & & & & & \\
\hline 3. Sciences & $\begin{array}{l}.48^{* *}[.26, . \\
63]\end{array}$ & $\begin{array}{l}.70^{* *}[.45, . \\
81]\end{array}$ & - & & & & \\
\hline 4. Langues & $\begin{array}{l}.39^{*}[.18, \\
56]\end{array}$ & $\begin{array}{l}.72^{* *}[.55, . \\
82]\end{array}$ & $\begin{array}{l}.88^{* *}[.83, . \\
92]\end{array}$ & - & & & \\
\hline $\begin{array}{l}\text { 5. Sciences } \\
\text { sociales }\end{array}$ & $\begin{array}{l}.39^{* *}[.18, . \\
56]\end{array}$ & $\begin{array}{l}.60^{* *}[.42, . \\
73]\end{array}$ & $\begin{array}{l}.82^{* *}[.73, . \\
88]\end{array}$ & $\begin{array}{l}.83^{* *}[.75, . \\
88]\end{array}$ & - & & \\
\hline 6. Arts & $\begin{array}{l}.34^{*}[.13, \\
52]\end{array}$ & $\begin{array}{l}.38^{*}[.14, . \\
54]\end{array}$ & $\begin{array}{l}.64^{* *}[.49, . \\
75]\end{array}$ & $\begin{array}{l}.61^{* *}[.46, . \\
73]\end{array}$ & $\begin{array}{l}.54^{* *}[.31, . \\
68]\end{array}$ & - & \\
\hline 7. MSG & $\begin{array}{l}.50^{* *}[.31, . \\
65]\end{array}$ & $\begin{array}{l}.70^{* *}[.48, . \\
80]\end{array}$ & $\begin{array}{l}.97^{* *}[.95, . . \\
98]\end{array}$ & $\begin{array}{l}.92^{* *}[.88, . \\
94]\end{array}$ & $\begin{array}{l}.87^{* *}[.80, . \\
92]\end{array}$ & $\begin{array}{l}.75^{* *}[.63, . \\
83]\end{array}$ & - \\
\hline Moyenne & 30.98 & 46.91 & 12.26 & 12.33 & 11.95 & 13.48 & 12.73 \\
\hline Skewness & .22 & -.33 & -.13 & -.36 & -.17 & -.03 & -.21 \\
\hline Kurtosis & -.30 & -.31 & -.27 & -.26 & -.62 & -.08 & .07 \\
\hline
\end{tabular}

Note. $\mathrm{N}=81$. Les $\mathrm{p}$-values ont été ajustées par la méthode de correction de Bonferronni. Entre crochets, l'intervalle de confiance à $95 \%$ a été estimé par une procédure bootstrap accélérée avec correction de biais de 1000 échantillons (Kelley, 2005 ; Thompson, 1993). Sciences: Mathématiques, Sciences physiques et chimiques et Science de la vie et de la terre; Langues : Français et Anglais ; Sciences sociales : Histoire et Géographie ; Arts : Arts plastiques et Éducation musicale; MSG: Moyenne scolaire générale au $1^{\text {er }}$ trimestre.

TABLE 2. Correlation between the average of the school grades and the BV9 and B53 scores 
TABLEAU 3. Régression multiple des performances scolaires en Sciences, Langues, Sciences sociales, Arts et des Moyennes scolaires générales sur les scores aux BV9 et B53

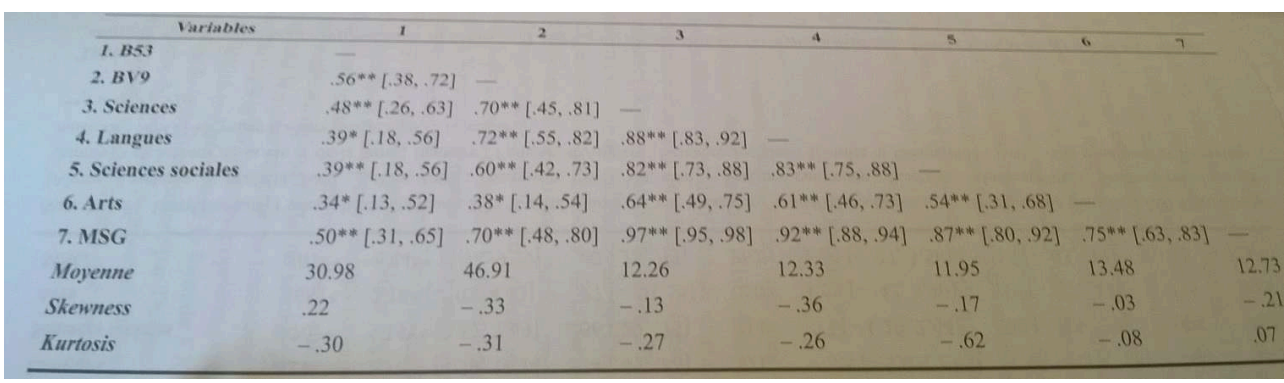
procédure bootstrap accélérée avec correction de biais de 1000 échantillons (Algina, Keselman, \& Penfield, 2007; Kelley, 2005; Thompson, 1993). Var. com. = Variance commune; Sciences : Mathématiques, Sciences physiques et chimiques et Science de la vie et de la terre; Langues: Français et Anglais; Sciences sociales: Histoire et Géographie; Arts: Arts plastiques et Éducation musicale; MSG : Moyenne scolaire générale au $1^{\text {er }}$ trimestre.

TABLE 3. Multiple regression school performances in Sciences, Languages, Social Science, Arts and Mean Scholastic General on BV9 and B53 scores

Dans l'ensemble, le BV9 expliquerait $26,2 \%$ de la variation de la moyenne scolaire générale alors que le B53 n'en explique que 1,5\%. L'interaction entre ces deux tests expliquerait $23 \%$ de la variation de la MSG.

L'analyse post hoc de puissance statistique (1- $\beta$ ) des différentes régressions multiples entre les performances scolaires dans les différentes disciplines et les deux tests est satisfaisante au regard du faible effectif. En effet, les puissances statistiques de nos régressions sont toutes supérieures à .90. Cela signifie donc que nous avons plus de $90 \%$ de chance de rejeter correctement l'hypothèse nulle, c'est-à-dire de considérer que les scores à nos deux tests n'influencent pas les performances scolaires. Par ailleurs, la taille des effets de nos prédicteurs va de modéré $\left(f^{2}=.20\right.$, en arts) à large $\left(f^{2}=1.09\right.$, en langues).

\section{Discussion}

À notre connaissance, peu d'études ont analysé la prédiction des notes scolaires obtenues dans diverses disciplines par l'évaluation conjointe de l'aptitude fluide (Gf) et de l'aptitude cristallisée (Gc). La majorité des recherches examine ce type de relation avec des tests standardisés des performances scolaires (Rindermann \& Neubauer, 2004). La notation scolaire peut être considérée comme un indicateur de performance scolaire, même si elle dépasse largement la mesure objective de la performance de l'enfant. En effet, les notes scolaires ont une variance, parfois importante, liée à la subjectivité de l'évaluateur (Chartier, 2010). En France, les notes scolaires sont importantes dans la décision du passage à l'année supérieure, surtout pour la classe de troisième. Nous nous sommes donc appuyés sur les évaluations "réelles » effectuées par les enseignants dans l'analyse du lien entre les aptitudes cognitives (Gc / Gf) et la

L'orientation scolaire et professionnelle, 45/3 | 2016 
réussite scolaire. Notre objectif est de comprendre le lien entre les tests utilisés et les performances scolaires observées. des études précédentes au sujet de la variance expliquée entre les performances scolaires et les aptitudes intellectuelles (Luo \& Petrill, 1999 ; Rohde \& Thompson, 2007). Par ailleurs, cela signifie qu'environ $50 \%$ de la variance de la moyenne scolaire générale reste inexpliquée par les aptitudes Gc et Gf. Cela suggère que cette variance inexpliquée peut être appréhendée par de nombreuses compétences cognitives et non cognitives (Veas, Castejón, Gilar, \& Miñano, 2015). Le sentiment d'efficacité personnel (Bandura, 1993; Bandura, Barbaranelli, Caprara, \& Pastorelli, 1996), l'estime de soi scolaire (Courtinat-Camps, 2010 ; Richardson, Abraham, \& Bond, 2012) et la motivation (Spinath, Spinath, Harlaar, \& Plomin, 2006 ; Steinmayr \& Spinath, 2008) affecteraient l'expérience et la réussite scolaire. Au niveau cognitif, certaines études ont montré l'importance de la mémoire de travail dans les apprentissages (Alloway \& Alloway, 2010 ; Barrouillet, Camos, Morlaix, \& Suchaut, 2008; Lépine, Barrouillet, \& Camos, 2005). Par ailleurs, le niveau socio-économique de la famille a également un fort impact sur les performances scolaires de l'enfant (Sirin, 2005). Tous ces facteurs devraient être donc pris en compte dans les études ultérieures et dans la pratique afin de mieux comprendre la réussite scolaire.

Une différence plus ou moins importante au niveau de la part d'explication apparaitt également selon les disciplines. Cette disparité interdisciplinaire peut aller d'environ $48 \%$ de la variance inexpliquée en langues à environ $83 \%$ en arts (voir tableau 3). Les aptitudes Gc et Gf sont impliquées dans toutes les matières. Cependant, elles ne le sont pas avec la même intensité. Nous observons que l'aptitude Gf serait plus liée aux disciplines dites scientifiques que celles dites littéraires. Cependant, nous remarquons une corrélation entre les notes en maths-sciences et l'aptitude Gc, qui paraît plus forte que celle avec l'aptitude Gf (voir tableau 2). Ce phénomène serait dû à l'accumulation de concepts propres au domaine scientifique (Beauducel et al., 2001). 
31 Nos résultats confirment l'idée selon laquelle la mesure des capacités intellectuelles est fortement liée aux performances scolaires (Roth et al., 2015). La taxonomie des capacités intellectuelles nous permet d'apporter une meilleure compréhension du lien entre la réussite scolaire et l'intelligence (McGrew \& Wendling, 2010). En effet, nous observons que l'aptitude Gc serait la plus impliquée dans la variance interindividuelle au niveau des notes scolaires. L'aptitude Gc serait donc un meilleur prédicteur de réussite scolaire que l'aptitude Gf chez les élèves (Colom, \& Flores-Mendoza, 2007; Postlethwaite, 2011). L'explication de cette supériorité serait que l'accumulation des connaissances aurait un plus fort impact sur l'apprentissage de nouvelles connaissances que le raisonnement fluide (Emmanuelli \& Weismann-Arcache, 2006).

Les capacités intellectuelles sont les meilleurs prédicteurs des performances scolaires. D'autres aptitudes cognitives, comme la mémoire de travail et les fonctions exécutives, et non cognitives, comme la motivation, le sentiment d'efficacité personnelle et l'image de soi, permettraient d'expliquer la variance résiduelle au niveau des performances scolaires. La "sous-réalisation» de certains élèves peut également expliquer ce pourcentage d'erreur de prédiction. La comparaison entre ces évaluations et les performances scolaires permettrait de mieux identifier les forces et les faiblesses de l'élève. Ces analyses permettent d'élaborer des moyens d'intervention en s'appuyant sur les points forts de l'élève. Par exemple, si l'élève a de faibles résultats scolaires, de bonnes capacités de raisonnement inductif (B53) et de faibles performances au BV9, alors cela indiquerait soit l'existence de facteurs empêchant le stockage ou le déploiement de connaissances chez l'élève, soit de faibles connaissances générales. Cela signifie que l'élève a du potentiel, avec cependant l'existence de facteurs empêchant la réalisation de ce potentiel. Ces facteurs devront être identifiés par l'élève avec l'aide du COP afin de trouver des stratégies pédagogiques.

Les résultats de notre étude doivent être interprétés avec précaution, car notre échantillon n'est pas totalement représentatif de l'ensemble des classes de troisième. En effet, l'échantillon de notre étude comporte deux classes composées d'élèves appartenant à la section européenne qui suivent des enseignements spécifiques d'approfondissement de la langue et de la culture anglaise. Il est donc difficile d'affirmer que ce type de classes soit représentatif de l'ensemble des classes de troisième. De nouvelles études avec un plus grand échantillon nécessiteraient d'être conduites afin de confirmer nos résultats.

\section{Conclusion}

Les tests BV9 et B53 sont utilisés, dans l'académie d'Amiens, pour comprendre le dynamisme intellectuel des élèves de troisième d'enseignement général. La présente recherche montre que ces deux tests semblent être des outils fiables. Pour les élèves de troisième générale, le test $\mathrm{BV} 9$ semble être un meilleur prédicteur des performances scolaires dans l'ensemble des disciplines que le test B53. Les performances à ces deux tests standardisés apportent donc des informations utiles au COP. Ils permettent de préciser la nature de la difficulté potentielle des élèves dans le domaine scolaire qui peut être dû à des difficultés au raisonnement ou à la capacité à accumuler de nouveaux concepts.

Ces évaluations constituent un maillon essentiel dans l'identification des élèves potentiellement fragilisés dans les apprentissages. Elles doivent être considérées 
comme un élément explicatif des performances scolaires parmi d'autres facteurs (cognitifs ou non cognitifs). Elles reflètent une estimation des aptitudes de raisonnement fluide et de compréhension-connaissance à un moment donné. L'interprétation doit se faire avec prudence. Ces outils peuvent contribuer à alimenter une réflexion sur le type de soutien le mieux adapté pour l'élève. Le rôle de ces évaluations n'est bien évidemment pas de dissuader l'élève dans son choix d'orientation, mais de mieux l'accompagner dans la voie qu'il aura choisie. L'identification des obstacles permet de trouver avec l'élève des moyens de les dépasser. Il est donc important de restituer les résultats à l'élève et à la famille en utilisant un vocable clair et compris de tous. Le rôle de l'accompagnement psychopédagogique est de comprendre et faire comprendre la raison de la distance éventuelle entre les performances scolaires et les capacités intellectuelles de l'élève.

\section{BIBLIOGRAPHIE}

Alloway, T. P. \& Alloway, R. G. (2010). Investigating the Predictive Roles of Working Memory and IQ in Academic Attainment. Journal of Experimental Child Psychology, 106(1), 20-29.

Ackerman, P. L., Beier, M. E., \& Boyle, M. O. (2005). Working Memory and Intelligence: The Same or Different Constructs? Psychological Bulletin, 131(1), 30-60.

Algina, J., Keselman, H. J., \& Penfield, R. D. (2007). Confidence Intervals for an Effect Size Measure in Multiple Linear Regression. Educational and Psychological Measurement, 67(2), 207-218.

Bandura, A. (1993). Perceived Self-Efficacy in Cognitive Development and Functioning. Educational Psychologist, 28(2), 117-148.

Bandura, A., Barbaranelli, C., Caprara, G. V., \& Pastorelli, C. (1996). Multifaceted Impact of SelfEfficacy Beliefs on Academic Functioning. Child Development, 67(3), 1206-1222.

Barrouillet, P., Camos, V., Morlaix, S., \& Suchaut, B. (2008). Progressions scolaires, mémoire de travail et origine sociale : quels liens à l'école élémentaire ? Revue Française de Pédagogie, 162, 5-14.

Beauducel, A., Brocke, B., \& Liepmann, D. (2001). Perspectives on Fluid and Crystallized Intelligence: Facets for Verbal, Numerical, and Figural Intelligence. Personality and Individual Differences, 30(6), 977-994.

Benson, N. (2008). Cattell-Horn-Carroll Cognitive Abilities and Reading Achievement. Journal of Psychoeducational Assessment, 26(1), 27-41.

Bernaud, J.-L. (2014). Méthodes de tests et questionnaires en psychologie. Paris : Dunod.

Bickley, P. G., Keith, T. Z., \& Wolfle, L. M. (1995). The Three-Stratum Theory of Cognitive Abilities: Test of the Structure of Intelligence across the Life Span. Intelligence, 20(3), 309-328.

Bonnardel, R. (1966). Test d'intelligence générale BV9. Paris : Presses Universitaires de France.

Bonnardel, R. (1967). Test d'intelligence non verbale - B53. Issy-les-Moulineaux : Éditions scientifiques et psychologiques. 
Canty, A. \& Ripley, B. (2015). boot: Bootstrap R (S-Plus) Functions (version 1.3-17). [Computer software].

Carroll, J. B. (1991). No Demonstration that $\mathrm{g}$ is not Unitary, but there's more to the Story: Comment on Kranzler and Jensen. Intelligence, 15(4), 423-436.

Carroll, J. B. (1993). Human Cognitive Abilities. Cambridge University Press.

Cattell, R. B. (1963). Theory of Fluid and Crystallized Intelligence: A Critical Experiment. Journal of Educational Psychology, 54(1), 1-22.

Ceci, S. J. (1991). How Much does Schooling Influence General Intelligence and its Cognitive Components? A Reassessment of the Evidence. Developmental Psychology, 27(5), 703-722.

Chartier, P. (2010). Évolution des notes scolaires entre le collège et le lycée : interprétations et enjeux, Mesure et évaluation en éducation, 33(3), 55-73.

Colom, R. \& Flores-Mendoza, C. E. (2007). Intelligence Predicts Scholastic Achievement Irrespective of SES Factors: Evidence from Brazil. Intelligence, 35(3), 243-251.

Cornoldi, C., Orsini, A., Cianci, L., Giofrè, D., \& Pezzuti, L. (2013). Intelligence and Working Memory Control: Evidence from the WISC-IV Administration to Italian Children. Learning and Individual Differences, 26(C), 9-14.

Courtinat-Camps, A. (2010). Socialisation familiale, estime de soi et expérience scolaire chez des collégien(ne)s à haut potentiel. Pratiques psychologiques, 16(4), 319-335.

Dang, C.-P., Braeken, J., Ferrer, E., \& Liu, C. (2012). Unitary or Non-Unitary Nature of Working Memory? Evidence from its Relation to General Fluid and Crystallized Intelligence. Intelligence, 40(5), 499-508.

Demetriou, A., Mouyi, A., \& Spanoudis, G. (2008). Modelling the Structure and Development of g. Intelligence, 36(5), 437-454.

Emmanuelli, M. \& Weismann-Arcache, C. (2006). Approche clinique des enfants à haut potentiel. In T. Lubart (éd.), Enfants exceptionnels. Rosny-sous-Bois : Bréal.

Flores-Mendoza, C., Mansur-Alves, M., Ardila, R., Rosas, R. D., Guerrero-Leiva, M. K., Maqueo, M. E. L.-G., Gallegos, M., Colareta, N. R., \& León, A. B. (2015). Fluid Intelligence and School Performance and its Relationship with Social Variables in Latin American Samples. Intelligence, 49, 66-83.

Friedman, N. P., Miyake, A., Corley, R. P., Young, S. E., DeFries, J. C., \& Hewitt, J. K. (2006). Not All Executive Functions Are Related to Intelligence. Psychological Science, 17(2), 172-179.

Gagné, F. \& St Père, F. S. (2002). When IQ is Controlled, does Motivation still Predict Achievement? Intelligence, 30(1), 71-100.

Gardner, H. (1983). Frame of Mind: The Theory of Multiple Intelligences. New York: Basic Books.

Gignac, G. E. (2006). Evaluating Subtest "g" Saturation Levels via the Single Trait-Correlated Uniqueness (STCU) SEM Approach: Evidence in Favor of Crystallized Subtests as the Best Indicators of " $\mathrm{g}$ ". Intelligence, 34(1), 29-46.

Golay, P., Reverte, I., Rossier, J., Favez, N., \& Lecerf, T. (2013). Further Insights on the French WISC-IV Factor Structure through Bayesian Structural Equation Modeling. Psychological Assessment, 25(2), 496-508.

Gustafsson, J. E. \& Balke, G. (1993). General and Specific Abilities as Predictors of School Achievement. Multivariate Behavioral Research, 28(4), 407-434. 
Horn, J. L. \& Blankson, N. (2005). Foundations for Better Understanding of Cognitive Abilities. In D. P. Flanagan \& P. L. Harrison (eds.), Contemporary Intellectual Assessment: Theories, Tests, and Issues (pp. 41-68). New York: Guilford Press.

Horn, J. L. \& Cattell, R. B. (1966). Refinement and Test of the Theory of Fluid and Crystallized General Intelligences. Journal of Educational Psychology, 57(5), 253-270.

Jensen, A. R. (1998). The g Factor: The Science of Mental Ability. Westport, CT: Praeger.

Juhel, J. (1993). Should we take the Shape of Reaction time Distributions into Account when Studying the Relationship between RT and Psychometric Intelligence? Personality and Individual Differences, 14(1), 293-296.

Kan, K.-J., Kievit, R. A., Dolan, C., \& Van der Maas, H. (2011). On the Interpretation of the CHC Factor Gc. Intelligence, 39(5), 292-302.

Kaufman, S. B., Reynolds, M. R., Liu, X., Kaufman, A. S., \& McGrew, K. S. (2012). Are Cognitive g and Academic Achievement $g$ One and the Same g? An Exploration on the Woodcock-Johnson and Kaufman Tests. Intelligence, 40(2), 123-138.

Keith, T. Z., Fine, J. G., Taub, G. E., Reynolds, M. R., \& Kranzler, J. H. (2006). Higher Order, Multisample, Confirmatory Factor Analysis of the Wechsler Intelligence Scale for Children: What does it Measure? School Psychology Review, 35(1), 108-127.

Kelley, K. (2005). The Effects of Nonnormal Distributions on Confidence Intervals Around the Standardized Mean Difference: Bootstrap and Parametric Confidence Intervals. Educational and Psychological Measurement, 65(1), 51-69.

Kuncel, N. R., Hezlett, S. A., \& Ones, D. S. (2004). Academic Performance, Career Potential, Creativity, and Job Performance: Can One Construct Predict Them All? Journal of Personality and Social Psychology, 86(1), 148-161.

Kvist, A. V. \& Gustafsson, J.-E. (2008). The Relation between Fluid Intelligence and the General Factor as a Function of Cultural Background: A Test of Cattell's Investment Theory. Intelligence, 36(5), 422-436.

Lépine, R., Barrouillet, P., \& Camos, V. (2005). What Makes Working Memory Spans so Predictive of High-Level Cognition? Psychonomic Bulletin \& Review, 12(1), 165-170.

Luo, D. \& Petrill, S. A. (1999). Elementary Cognitive Tasks and their Roles in g Estimates. Intelligence, 27(2), 157-174.

Mackintosh, N. J. (2004). Q.I. \& intelligence humaine (A. Brossard). Bruxelles : De Boeck.

McGrew, K. S. (2009). CHC Theory and the Human Cognitive Abilities Project: Standing on the Shoulders of the Giants of Psychometric Intelligence Research. Intelligence, 37(1), 1-10.

McGrew, K. S. \& Wendling, B. J. (2010). Cattell-Horn-Carroll Cognitive-Achievement Relations: What we have Learned from the Past 20 Years of Research. Psychology in the Schools, 47(7), 652-675. Newton, J. H. \& McGrew, K. (2010). Introduction to the Special Issue: Current Research in CattellHorn-Carroll-Based Assessment. Psychology in the Schools, 47(7), 621-634.

Nicollet, J., Guillen, C. J., Jouhar, A.-C., \& Rossier, J. (2009). Performance aux tests d'intelligence : vers une inversion de l'effet Flynn? L'Orientation scolaire et professionnelle, 38(3), 353-368.

Nimon, K., Oswald, F., \& Roberts, K. J. (2013). yhat: Interpreting Regression Effects (version 2.0-0). [Computer software]. Retrieved from http://CRAN.R-project.org/package=yhat 
Nimon, K., Lewis, M., Kane, R., \& Haynes, R. M. (2008). An R Package to Compute Commonality Coefficients in the Multiple Regression Case: An Introduction to the Package and a Practical Example. Behavior Research Methods, 40(2), 457-466.

Parkin, J. R. \& Beaujean, A. A. (2012). The Effects of Wechsler Intelligence Scale for ChildrenFourth Edition Cognitive Abilities on Math Achievement. Journal of School Psychology, 50(1), 113-128.

Postlethwaite, B. E. (2011). Fluid Ability, Crystallized Ability, and Performance Across Multiple Domains: a Meta-Analysis. Doctoral dissertation. Retrieved from http://ir.uiowa.edu/etd/1255

R Core Team. (2015). R: A Language and Environment for Statistical Computing. Vienna, Austria: R Foundation for Statistical Computing. Retrieved from http://www.R-project.org

Revelle, W. (2015). psych: Procedures for Personality and Psychological Research (version 1.5.8). [Computer software]. Evanston, Illinois. Retrieved from http://CRAN.R-project.org/ package=psych

Reynolds, M. R., Floyd, R. G., \& Niileksela, C. R. (2013). How well is Psychometric g Indexed by Global Composites? Evidence from Three Popular Intelligence Tests. Psychological Assessment, 25(4), 1314-1321.

Reynolds, M. R., Keith, T. Z., Flanagan, D. P., \& Alfonso, V. C. (2013). A Cross-Battery, Reference Variable, Confirmatory Factor Analytic Investigation of the CHC Taxonomy. Journal of School Psychology, 51(4), 535-555.

Richardson, M., Abraham, C., \& Bond, R. (2012). Psychological Correlates of University Students' Academic Performance: A Systematic Review and Meta-Analysis. Psychological Bulletin, 138(2), 353-387.

Rindermann, H. \& Neubauer, A. (2004). Processing Speed, Intelligence, Creativity, and School Performance: Testing of Causal Hypotheses using Structural Equation Models. Intelligence, 32(6), 573-589.

Rindermann, H., Flores-Mendoza, C., \& Mansur-Alves, M. (2010). Reciprocal Effects between Fluid and Crystallized Intelligence and their Dependence on Parents' Socioeconomic Status and Education. Learning and Individual Differences, 20(5), 544-548.

Rohde, T. E. \& Thompson, L. A. (2007). Predicting Academic Achievement with Cognitive Ability. Intelligence, 35(1), 83-92.

Roth, B., Becker, N., Romeyke, S., Schäfer, S., Domnick, F., \& Spinath, F. M. (2015). Intelligence and School Grades: A Meta-Analysis. Intelligence, 53, 118-137.

Roth, P. L., Bevier, C. A., Switzer, F. S., \& Schippmann, J. S. (1996). Meta-Analyzing the Relationship between Grades and Job Performance. Journal of Applied Psychology, 81(5), 548-556.

Schipolowski, S., Wilhelm, O., \& Schroeders, U. (2014). On the Nature of Crystallized Intelligence: the Relationship between Verbal Ability and Factual Knowledge. Intelligence, 46, 156-168.

Schneider, W. J. (2013a). Principles of Assessment of Aptitude and Achievement. In D. H., Saklofske, C. R. Reynolds, \& V. L. Schwean (eds.), The Oxford Handbook of Child Psychological Assessment. New York: Oxford University Press.

Schneider, W. J. (2013b). What if We Took our Models Seriously? Estimating Latent Scores in Individuals. Journal of Psychoeducational Assessment, 31(2), 186-201. 
Schneider, W. J. \& McGrew, K. S. (2012). The Cattell-Horn-Carroll Model of Intelligence. In D. P. Flanagan \& P. L. Harrison (eds.), Contemporary Intellectual Assessment Theories, Tests, and Issues (3rd ed., pp. 99-144). New York: Guilford Press.

Sirin, S. R. (2005). Socioeconomic Status and Academic Achievement: A Meta-Analytic Review of Research. Review of Educational Research, 75(3), 417-453.

Spearman, C. (1904). "General Intelligence," Objectively Determined and Measured. The American Journal of Psychology, 15(2), 201-293.

Spinath, B., Spinath, F. M., Harlaar, N., \& Plomin, R. (2006). Predicting School Achievement from General Cognitive Ability, Self-Perceived Ability, and Intrinsic Value. Intelligence, 34(4), 363-374.

Steinmayr, R. \& Spinath, B. (2008). The Importance of Motivation as a Predictor of School Achievement. Learning and Individual Differences, 19(1), 80-90.

Taub, G. E., Keith, T. Z., Floyd, R. G., \& McGrew, K. S. (2008). Effects of General and Broad Cognitive Abilities on Mathematics Achievement. School Psychology Quarterly, 23(2), 187-198.

Thiébaut, E. (2000). Les tests de raisonnement, B53-C, BLS4-C. Paris : Éditions et Applications psychologiques.

Van Der Maas, H. L. J., Dolan, C. V., Grasman, R. P. P. P., Wicherts, J. M., Huizenga, H. M., \& Raijmakers, M. E. J. (2006). A Dynamical Model of General Intelligence: The Positive Manifold of Intelligence by Mutualism. Psychological Review, 113(4), 842-861.

Veas, A., Castejón, J.-L., Gilar, R., \& Miñano, P. (2015). Academic Achievement in Early Adolescence: The Influence of Cognitive and Non-Cognitive Variables. The Journal of General Psychology, 142(4), 273-294.

Warne, R. T. (2016). Five Reasons to Put the g Back Into Giftedness: An Argument for Applying the Cattell-Horn-Carroll Theory of Intelligence to Gifted Education Research and Practice. Gifted Child Quarterly, 60(1), 3-15.

Walberg, H. J. (1984). Improving the Productivity of America's Schools. Educational Leadership, 19-27.

\section{NOTES}

1. Cela correspond a un intervalle de confiance à $95 \%$.

\section{RÉSUMÉS}

Dans certaines Académies françaises, les tests B53 et BV9 sont utilisés dans le cadre de l'orientation scolaire en troisième générale. Ils permettraient d'avoir respectivement une estimation des capacités de raisonnement fluide (Gf) et de compréhension-connaissance (Gc). L'objectif de cette étude est de comprendre le fonctionnement cognitif global de l'élève par rapport à ses performances scolaires. Nos résultats montrent que le BV9 semble être un meilleur prédicteur des performances scolaires que le B53 dans l'ensemble des disciplines. Les deux tests 
expliquent environ $50 \%$ de la variance de la moyenne scolaire générale (MSG). L'interprétation et les limites de ces résultats sont discutés.

In the many french Academy, the B53 and BV9 tests are used as part of the school counseling in $9^{\text {th }}$ grade. They would estimate respectively the abilities of fluid reasoning (Gf) and comprehension-knowledge (Gc). The objective of this study is to understand the overall cognitive functioning of the student in relation to his academic performance. Our results show that the BV9 seems to be a better predictor of school performance than the B53 in many disciplines. Both tests explain about $50 \%$ of the variance in overall academic average (MSG). The interpretation and limits of the findings are discussed.

\section{INDEX}

Keywords : Fluid reasoning, comprehension-knowledge, academic achievement, BV9, B53

Mots-clés : raisonnement fluide, compréhension-connaissance, performances scolaires, BV9, B53

\section{AUTEURS}

\section{ALEXANDRE AUBRY}

Doctorant en psychologie du développement, Université de Picardie Jules Verne. Thème de recherche : psychologie du développement. Contact : Université de Picardie Jules Verne, Chemin du Thil, 80000 Amiens. Courriel : alexandre.aubry@etud.u-picardie.fr

\section{BÉATRICE BOURDIN}

Maîtresse de conférences HDR en psychologie du développement, Université de Picardie Jules Verne 